\title{
PRÉLÈVEMENTS FISCAUX ET PRESTATIONS SOCIALES EN BELGIQUE : L'IMPACT DE VINGT ANNÉES DE RÉFORMES
}

André Decoster,Sergio Perelman,Dieter Vandelannoote,Toon Vanheukelom, Gerlinde Verbist

\section{Revue française d'économie | « Revue française d'économie »}

2014/4 Volume XXIX | pages 87 à 127

ISSN 0769-0479

Article disponible en ligne à l'adresse :

http://www.cairn.info/revue-francaise-d-economie-2014-4-page-87.htm

\section{Pour citer cet article :}

André Decoster et al., «Prélèvements fiscaux et prestations sociales en Belgique : l'impact de vingt années de réformes », Revue française d'économie 2014/4 (Volume XXIX), p. 87-127. DOI 10.3917/rfe.144.0087

Distribution électronique Cairn.info pour Revue française d'économie.

(C) Revue française d'économie. Tous droits réservés pour tous pays.

La reproduction ou représentation de cet article, notamment par photocopie, n'est autorisée que dans les limites des conditions générales d'utilisation du site ou, le cas échéant, des conditions générales de la licence souscrite par votre établissement. Toute autre reproduction ou représentation, en tout ou partie, sous quelque forme et de quelque manière que ce soit, est interdite sauf accord préalable et écrit de l'éditeur, en dehors des cas prévus par la législation en vigueur en France. Il est précisé que son stockage dans une base de données est également interdit. 


\section{André DECOSTER Sergio PERELMAN Dieter VANDELANNOOTE Toon VANHEUKELOM Gerlinde VERBIST}

Prélèvements fiscaux et prestations sociales en Belgique : l'impact de vingt années de réformes

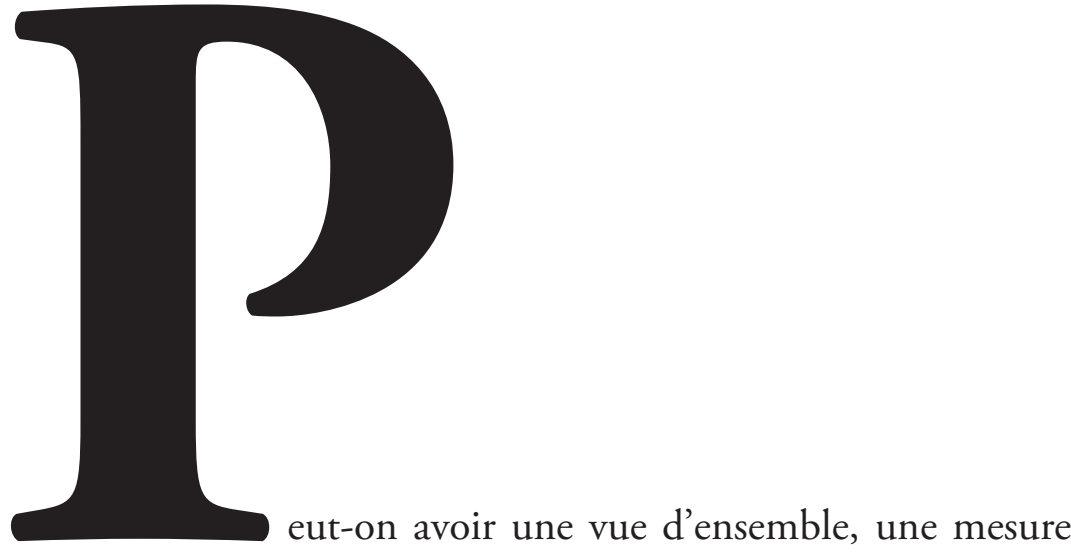
synthétique, de l'effet des réformes fiscales et sociales sur l'équité et l'efficacité ? C'est la question à laquelle nous essaierons de répondre dans cet article en prenant comme exemple le cas de la Belgique au cours des deux dernières décennies. Une série de 
réformes importantes en matière de prélèvements fiscaux, y compris les cotisations sociales personnelles, et de prestations sociales ont eu lieu au cours de cette période, motivées par des objectifs d'équité ou d'efficacité économique, avec des effets à la fois sur le revenu disponible des ménages et sur le taux marginal d'imposition du travail. Parce que ces réformes affectent différemment les diverses catégories de la population, il n'est pas aisé d'en mesurer l'impact. Pour répondre à cette difficulté, se sont peu à peu développées des techniques de micro-simulation basées sur des données individuelles, de source administrative ou d'enquêtes, combinées avec des méthodes d'estimation économétrique.

D'une part, le travail de Bargain et Callan [2010] propose l'utilisation de techniques de micro-simulation dynamique basées sur le calcul de distributions de revenus contrefactuelles pour la mesure des effets des réformes sur l'équité, d'autre part, basés sur les mêmes techniques, les travaux de Kleven et Kreiner [2006] proposent une mesure résumant l'impact des réformes, au travers de leurs effets sur les incitants financiers, influençant l'offre de travail. Cette mesure, censée représenter le coût marginal des fonds publics (MCPF), fut initialement calculée sur base d'indicateurs macroéconomiques ou d'agents représentatifs et, souvent, limitée aux systèmes de taxation proportionnels, comme dans Browning [1976]. Ce sont Hansson et Stuart [1985] et Dahlby [1998], parmi d'autres, qui ont étendu ce concept à l'étude des changements marginaux dans des systèmes de taxation progressifs par paliers. Enfin, Kleven et Kreiner [2006] ont incorporé dans le MCPF les effets induits sur la participation au marché du travail, la marge extensive, considérée à partir des travaux de Saez [2002] comme l'élément essentiel à prendre en compte pour étudier les distorsions introduites par la politique fiscale ${ }^{1}$.

Dans cet article nous suivons les travaux de Bargain et Callan afin de mesurer l'effet des réformes du système fiscal belge sur l'équité, et nous adoptons la définition étendue du MCPF proposée par Kleven et Kreiner comme mesure d'efficacité.

Le cas de la Belgique ne diffère pas de celui d'autres paysmembres de l'OCDE qui, au cours des vingt dernières années, ont mené des réformes importantes aussi bien dans leur système de 
taxation que dans celui des transferts sociaux. Par le passé, la plupart des études se sont intéressé à différents aspects des réformes de manière isolée, par exemple Decoster et Van Camp [2001] ou Decoster et al [2012]. Dans cet article nous étudions l'ensemble des prélèvements, cotisations sociales et impôt direct sur le revenu, ainsi que les transferts sociaux sous la forme de revenus de remplacement, touchant les ménages belges. La période que nous étudions s'étend de 1992 à 2012 et, afin de mesurer l'effet des réformes, nous avons recours au modèle de micro-simulation Mefisto-Euromod ${ }^{2}$. Les données de base - caractéristiques individuelles et du ménage, source et montant des revenus, situation sur le marché du travail, ... - sont collectées dans le cadre de l'enquête européenne EU-SILC (Enquête sur les revenus et les conditions de vie) en 2010, dont les informations utilisées ici, notamment sur les revenus, se rapportent à l'année 2009. Notre but, dans cette étude, est d'analyser un ensemble de situations contrefactuelles. Pour être plus précis, la question que nous posons est : quel serait l'impact sur le revenu disponible des ménages, tel qu'observé en 2009, si l'ensemble des prélèvements fiscaux et des prestations sociales étaient calculés sur base des règles correspondant à une autre année. Comme nous le montrerons dans la section méthodologique qui suit, cette manière de procéder a comme avantage d'isoler l'effet des réformes proprement dites des effets dus à l'évolution dans la structure de la population et aux changements dans le niveau et la composition du revenu brut (avant prélèvements et transferts). Les années choisies pour cette analyse contrefactuelle sont 1992, 2001, 2007 et 2012.

Une remarque préliminaire concerne l'interprétation potentiellement erronée que l'on pourrait faire des résultats présentés ici. En effet, on serait tenté de considérer ces résultats comme la description d'un potentiel arbitrage équité-efficacité auquel les responsables auraient fait face à chaque moment. Notre objectif n'est justement pas de décrire cet arbitrage éventuel, ni les choix que ces responsables auraient faits dans ce contexte. La raison est que nous utilisons nos deux mesures agrégées, l'effet redistributif du système fiscal et l'effet de ce système sur les incitations de l'offre de travail, comme de simples outils descriptifs. En dehors du fait 
qu'ils sont à l'origine calculés à partir d'un même système fiscal, les deux mesures sont sans rapport direct. Les effets redistributifs sont calculés comme le résultat d'un système donné, et ne sont donc pas en rapport direct avec les changements marginaux survenus dans ce même système. De plus, le MCPF décrit ce même système en considérant un seul changement parmi toute une série d'éventuels changements marginaux. En ce sens, cet article diffère fondamentalement d'autres travaux publiés dans la littérature, en commençant par celui de Browning et Johnson [1984], mais également celui de Mayshar [1990] et Dahlby [1998] qui quantifient de manière explicite l'arbitrage équité-efficacité à la marge. Pour y arriver, ces auteurs simulent le changement d'un élément spécifique du système de taxation, lequel correspond à un changement marginal bien identifié dans la progressivité de l'impôt (le revenu minimum garanti dans Browning et Johnson [1984], ou l'un des taux marginaux dans une taxe sur le revenu chez Dahlby [1998]), et calculent ensuite le MCPF. Dans ce cas, le MCPF mesure de manière explicite le coût en termes de bien-être de cette redistribution marginale $\mathrm{du}$ revenu ${ }^{3}$. Ce n'est pas ce que nous présentons ici. Nous partons de l'idée que les diverses réformes introduites dans le système fiscal belge ont affecté les incitations financières au travail de manière différente pour différents groupes dans la population ; ici répartis par décile de revenu. Nous utilisons simplement le MCPF comme une mesure sophistiquée d'agrégation de ces incitations, produites par le système fiscal, au sein d'une population hétérogène.

Après avoir présenté la méthodologie générale de l'étude, la section suivante sera consacrée à la présentation des principales réformes et la troisième section aux résultats des microsimulations. La dernière section contient les conclusions.

\section{Méthodologie}

Afin d'étudier la manière dont les réformes successives ont affecté le revenu disponible des ménages belges au cours de la période 
1992-2012 nous appliquons une version simplifiée de l'approche contrefactuelle proposée par Bargain et Callan [2010]. Elle consiste à simuler le revenu disponible pour les années 1992, 1997, 2001 et 2012, en appliquant les règles du système fiscal en vigueur pour ces années, tout en maintenant inchangées les caractéristiques des ménages, y compris le revenu qu'ils tirent du marché (hors taxes et transferts), de 2009 (EU-SILC [2010]). Sur base de ces distributions contrefactuelles du revenu disponible, nous calculons les propriétés redistributives du système fiscal. C'est sur la base de ces mêmes estimations que nous calculons les taux marginaux effectifs, marge extensive et marge intensive afin d'estimer les incitants financiers sur l'offre de travail.

\section{Distribution de revenus contrefactuelle}

Nous considérons le système fiscal de l'année $i$ comme une fonction qui, pour chaque individu $h$ disposant d'une série de revenus avant taxes et avant transferts et d'une série de caractéristiques sociodémographiques (âge, sexe, taille du ménage...) observées l'année $k$ et représentées par les vecteurs $y_{h}^{k}$ et $z_{h}^{k}$, respectivement, donne comme résultat, en forme scalaire, le revenu disponible $x_{h}^{k, i}$ :

$$
x_{b}^{k, i}=d^{i}\left(p^{i}, y_{h}^{k}, z_{b}^{k}\right)
$$

où $k$ indique l'année pour laquelle le revenu et les caractéristiques sociodémographiques sont observés et $i$, l'année pour laquelle le système fiscal est simulé. Le vecteur $p^{i}$ indique l'ensemble des paramètres du système : le montant des allocations familiales, les bornes inférieures et supérieures des tranches du revenu taxable, des allocations minima et maxima, le revenu à ne pas dépasser pour pouvoir bénéficier d'une allocation sociale de base (earning test), parmi d'autres. Toutes les autres caractéristiques du système fiscal sont capturées par la fonction $d^{i}($.). Dans notre application, les résultats sont exprimés en termes du revenu disponible par unité de consommation. A cet effet, nous 
utilisons l'échelle d'équivalence préconisée par l'OCDE, laquelle assigne la valeur 1,0 pour le premier adulte dans le ménage, 0,7 pour les autres adultes et 0,5 pour les enfants (personnes âgées de moins de 14 ans).

En faisant l'hypothèse que nous disposons de deux observations du revenu disponible, celles correspondant aux années 1 et $0, x_{h}^{1,1}$ et $x_{h}^{0,0}$, respectivement. Il est clair, à partir de l'équation (1), que le changement observé dans le revenu disponible peut provenir de différentes sources potentielles : le système fiscal, les revenus (avant taxes et transferts) ou bien les caractéristiques sociodémographiques. En nous servant du cadre conceptuel proposé par Bargain et Callan [2010], nous décomposons ce changement de la manière suivante :

$$
\begin{aligned}
\Delta x_{b} \equiv x_{b}^{1,1}-x_{b}^{0,0} & =d^{1}\left(p^{1}, y_{b}^{1}, z_{b}^{1}\right)-d^{0}\left(p^{0}, y_{b}^{0}, z_{b}^{O}\right) \\
& =\underbrace{}_{\begin{array}{c}
\text { effets des réformes } \\
d^{1}\left(p^{1}, y_{b}^{1}, z_{b}^{1}\right)-d^{0}\left(\alpha^{0,1} p^{0}, y_{b}^{1}, z_{b}^{I}\right)
\end{array}} \\
& +\underbrace{d^{0}\left(\alpha^{0,1} p^{0}, y_{b}^{1}, z_{b}^{1}\right)-d^{0}\left(\alpha^{0,1} p^{0}, \alpha^{O, 1} y_{b}^{O}, z_{b}^{O}\right)}_{\begin{array}{c}
\text { changement dans les revenus et dans } \\
\text { les caractéristiques sociodémographiques }
\end{array}} \\
& +\underbrace{d^{0}\left(\alpha^{0,1} p^{0}, \alpha^{O, 1} y_{b}^{O}, z_{b}^{O}\right)-d^{0}\left(p^{0}, y_{b}^{O}, z_{b}^{O}\right)}_{\text {accroissement de la charge fiscale }}
\end{aligned}
$$

où nous utilisons le scalaire $\alpha^{0,1}$ pour indiquer le changement dans les niveaux de prix (nominaux) entre les périodes 0 et 1 . Ainsi, le troisième terme de l'équation capture l'effet sur le revenu disponible résultant de l'inflation, l'indice de prix $\alpha^{0,1}$, avec la variation, au même taux, de l'ensemble de paramètres du système. Si le système est protégé contre l'inflation, ce terme devrait être égal à zéro. Autrement, en cas de non-linéarités dans le système, il y aura un accroissement de la charge fiscale. Le deuxième terme capture l'effet des changements dans les revenus du marché et dans les caractéristiques sociodémographiques et le premier terme permet d'isoler l'effet des changements dans le système fiscal. La manière dont ce terme est calculé indique que l'effet des réformes est estimé sur 
base des revenus et des caractéristiques observées à la période 1. En adoptant $\alpha^{0,1} p^{0}$ comme argument de $d^{0}($.) dans le premier terme, nous faisons l'hypothèse explicite que par défaut, en cas de nonréforme du système, il y aura une indexation parfaite de tous les paramètres du système. Mais une réforme peut aussi consister en l'absence d'indexation de certains paramètres, comme cela a été le cas dans les années 1990 en Belgique pour certains seuils de l'impôt sur le revenu des personnes ${ }^{4}$. Nous avons utilisé l'indice de prix à la consommation (voir tableau $\mathrm{n}^{\circ} 1$ ) pour tenir compte des changements dans les prix 5 .

Notre attention se portera ici sur le premier élément de l'équation (2), c'est-à-dire l'effet des réformes dû exclusivement au changement des paramètres du système. Les revenus bruts du marché et les caractéristiques socioéconomiques resteront inchangés tout au long de l'exercice de micro-simulation et seront ceux correspondant à l'année 2009 (EU-SILC [2010]). Malheureusement nous ne disposons pas, dans le cas de la Belgique, de bases de données comparables pour l'ensemble de la période (1992 à 2012) ${ }^{6}$. Comme le montrent Bargain et Callan [2010] le choix de l'année de base est important dans la mesure où il détermine la structure sociodémographique qui est appliquée pour l'ensemble des années. Néanmoins, ceci n'empêche pas de considérer le premier terme de l'équation (2) comme un indicateur fiable de l'effet des changements dans un système fiscal très complexe par définition ${ }^{7}$.

Comme indiqué précédemment, nous résumons l'effet des changements dans le système fiscal belge sur la période 19922012, en choisissant quatre années : 1992, 2001, 2007 et 2012. Le système en vigueur pour chacune de ces années est appliqué aux données sur les revenus des ménages de 2009 (EU-SILC [2010]), en faisant usage du modèle de microsimulation Mefisto-Euromod.

Ce modèle tient compte des compétences à la fois du gouvernement fédéral et des régions belges. Il simule de manière assez complète l'impôt sur le revenu des personnes physiques (IPP), les cotisations sociales, les allocations familiales et celles de l'assistance sociale. A cet effet, il paramétrise la structure du système de taxation et revalorise les montants nominaux appliqués chaque année. 
Il est important de remarquer que, de manière générale, il n'est pas possible de simuler des changements dans le montant attendu des pensions et des allocations de chômage sans disposer d'informations rétrospectives sur la carrière professionnelle des individus, comme c'est le cas des données transversales de EU-SILC utilisées ici. C'est la raison pour laquelle ces allocations ne sont pas simulées directement par Mefisto-Euromod.

Néanmoins, des réformes ont eu lieu pendant la période couverte par cette étude, réformes qui ont affecté de manière significative le montant des pensions et des allocations de chômage. Afin d'en tenir compte, nous avons fait le choix d'une méthode de simulation qui mime - dans la mesure du possible - les effets de ces réformes. Dans le cas des pensions, comme indiqué plus haut, les réformes ont consisté principalement dans l'adaptation au bien-être des montants des allocations pour des catégories bien définies de bénéficiaires, celles détaillées dans l'annexe $n^{\circ} 2$.

Nous avons appliqué ces adaptations en partant du scénario de base, en 2009. Quand un individu est observé en tant que bénéficiaire d'une pension en 2009, nous simulons le montant de l'allocation dans le cas où l'adaptation au bienêtre n'aurait pas eu lieu - situation contrefactuelle - aussi bien pour les années 1992, 2001 et 2007 comme pour l'année 2012. L'évolution du montant moyen de la pension en termes réels est celle présentée dans le tableau $\mathrm{n}^{\circ} 1^{8}$.

Pour les allocations de chômage nous avons procédé de la même manière. Nous avons estimé le taux de croissance moyen, en termes réels, des montants minimum et maximum des allocations, suite aux adaptations au bien-être, en faisant la distinction entre les personnes qui étaient au chômage depuis moins d'un an et celles qui l'étaient depuis plus longtemps. Ces taux sont présentés également au tableau $\mathrm{n}^{\circ} 1$. Comme dans le cas des pensions, cette façon de procéder est une simplification ; elle néglige les changements éventuels intervenus entre-temps dans les critères d'éligibilité.

Enfin, sur le tableau $\mathrm{n}^{\circ} 1$ nous rapportons également l'indice de croissance annuel du salaire réel au cours des différentes périodes. Il est clair que les différences observées entre ces taux de croissance et 
le taux d'adaptation au bien-être des allocations de chômage et des pensions, vont jouer un rôle important dans l'évaluation de l'effet des réformes, comme nous le constaterons plus loin. Il est aussi important de signaler ici que certains réformes, notamment celles qui concernent les allocations pour cause de maladie et invalidité, sont omises en raison du manque d'informations pour modéliser de manière suffisamment détaillée et fiable leurs effets.

\section{Tableau 1}

\section{Inflation, adaptation au bien-être et taux de croissance du salaire réel}

\begin{tabular}{|llllll|}
\hline \multicolumn{5}{|c|}{ Indice $(2009=100)$} \\
\hline & 1992 & 2001 & 2007 & 2009 & 2012 \\
\hline Prix à la consommation & 71,7 & 83,7 & 94,5 & 100,0 & 107,7 \\
\hline Salaire réel & 92,2 & 97,0 & 99,7 & 100,0 & 98,7 \\
\hline Adaptation au bien-être & & & & & \\
Pensions & 96,1 & 96,1 & 98,0 & 100,0 & 104,0 \\
Chômage ( $\leq 12$ mois) & 86,8 & 86,4 & 91,9 & 100,0 & 99,4 \\
Chômage (>12 mois) & 86,4 & 87,5 & 97,0 & 100,0 & 99,4 \\
\hline
\end{tabular}

\begin{tabular}{|lcccc|}
\hline \multicolumn{5}{|c|}{ Taux de croissance annuel (\%) } \\
\hline & $1992-$ & $2001-$ & $2007-$ & $1992-$ \\
& 2001 & 2007 & 2012 & 2012 \\
\hline Prix à la consommation & 1,73 & 2,04 & 2,65 & 2,06 \\
\hline Salaire réel & 0,57 & 0,46 & $-0,20$ & 0,34 \\
\hline $\begin{array}{l}\text { Adaptation au bien-être } \\
\text { Pensions }\end{array}$ & 0,00 & 0,33 & 1,20 & 0,40 \\
Chômage ( $\leq 12$ mois) & $-0,05$ & 1,03 & 1,58 & 0,68 \\
Chômage (>12 mois) & 0,14 & 1,73 & 0,49 & 0,70 \\
\hline
\end{tabular}

Source: Belgostat (Banque nationale de Belgique, statistiques) et SPF Sécurité sociale.

Afin de pouvoir comparer sur un diagramme simple les mesures synthétiques d'équité et d'efficacité, et pour des raisons de simplicité, nous avons choisi comme mesure d'équité l'indice de Reynolds-Smolensky, et comme mesure d'efficacité l'indice 
du coût marginal des fonds publics (MCPF). Le calcul de ces mesures est présenté ci-après.

\section{Mesure synthétique d'équité}

L'équation (3) présente l'indice de Reynolds-Smolensky, $\Pi^{R S}$, choisi pour mesurer l'effet redistributif du système fiscal. Ce coefficient mesure le changement dans la distribution du revenu disponible résultant du système fiscal. Une particularité de cet index est qu'il est décomposable. D'une part, le terme $t /(1-t)$, qui représente le taux moyen de taxation et, d'autre part, l'indice de Kakwani $\Pi^{K}$, qui mesure la disproportionalité du système fiscal réel par rapport à la situation hypothétique d'un système fiscal parfaitement proportionnel ${ }^{9}$ :

$$
\Pi^{R S}=\left(\frac{t}{1-t}\right) \cdot \Pi^{K}
$$

Afin d'analyser l'effet des réformes sur l'équité de la manière la plus générale, c'est-à-dire pour l'ensemble de la population, notre mesure est basée sur le revenu disponible du ménage. Par ailleurs, nous présenterons les effets en points de pourcentage de pouvoir d'achat par rapport au revenu disponible du ménage pour chacun des déciles de la population, distribuée elle-même en fonction du revenu équivalent par unité de consommation. Le revenu disponible du ménage est défini comme étant égal au revenu brut - salaires, revenus du capital, y compris revenus de la propriété, de pensions privées et de l'entreprise, plus transferts entre ménages - moins contributions sociales et taxes directes, et transferts sociaux, en plus.

\section{Mesure synthétique d'efficacité}

Comme indiqué précédemment, nous faisons le choix de mesurer l'impact des réformes du système fiscal (prélèvements-transferts), en calculant la variation observée dans le coût marginal des fonds 
publics (MCPF). Cette mesure donne une estimation du coût en termes de bien-être résultant de la distorsion additionnelle créée par un instrument fiscal utilisé pour lever un euro additionnel. Sur le plan empirique, Kleven et Kreiner [2006], à l'instar de Saez [2002], ont étendu ce concept, originellement limité à un effet sur l'intensité de l'offre de travail (marge intensive), avec la prise en compte de l'effet sur le taux d'emploi (marge extensive) ainsi que l'hétérogénéité des situations dans la population.

Dans ce qui suit, nous présentons tout d'abord le calcul de l'effet des taux marginaux de taxation, d'une part sur le taux de participation $\tau_{i}$ (marge extensive) et, d'autre part, sur l'intensité de l'offre de travail $m_{i}$ (taux marginal effectif, ou marge intensive), où $i$ indique la population par décile de revenu brut du travail $(i=1, \ldots 10)$. Ensuite nous définissons le coût marginal de fonds publics (MCPF), une mesure qui, comme le démontrent Kleven et Kreiner [2006], dépend des valeurs de $\tau_{i}$ et $m_{i}$ et des élasticités individuelles respectives, $\eta_{i}$ au niveau de la marge extensive et $\varepsilon_{i}$ au niveau de la marge intensive. Ces élasticités représentent dès lors les comportements attendus sur le marché du travail.

\section{Taux marginal de taxation: marge extensive}

Le taux marginal de taxation sur la participation au marché du travail (marge extensive), est une mesure de l'utilité associée à l'emploi par opposition au non-emploi. Dans quelle mesure le revenu brut de l'activité sera-t-il taxé si la personne participe effectivement au marché du travail, de manière explicite, au travers des taxes directes et de contributions sociales, ou de manière implicite, au travers de la perte de bénéfices ou transferts sociaux ? Si son revenu disponible est actuellement inférieur ou à peine plus élevé que dans la situation où elle ne travaillerait pas, elle est confrontée à une trappe la menant probablement à rester inactive.

L'équation (4) représente la contrainte budgétaire de l'individu $i$ en cas d'emploi. Elle met en rapport le revenu disponible $c_{i}^{E}$ avec le revenu brut du travail $w_{i} h$, où $h$ indique les heures de travail payées au salaire $w_{i}, T\left(w_{i} h\right)$ l'ensemble des 
taxes et de transferts en relation avec le revenu du travail, et $I_{i}$ le revenu hors travail ${ }^{10}$ :

$$
c_{i}^{E}=w_{i} h-T\left(w_{i} h\right)+I_{i}
$$

En cas de non-emploi, le revenu disponible est déterminé par le revenu hors travail et par d'éventuels transferts $T(0)$ :

$$
c_{i}^{N}=T(0)+I_{i}
$$

Donc, le taux marginal de taxation en cas d'emploi (marge extensive) est calculé comme suit :

$$
\tau_{i}=1-\frac{c_{i}^{E}-c_{i}^{N}}{w_{i} h}=\frac{T\left(w_{i} h\right)-T(0)}{w_{i} h}
$$

Le taux $\tau_{i}$ peut ensuite être décomposé en faisant la distinction entre les différents prélèvements et transferts :

$$
\boldsymbol{\tau}_{i}=\frac{S_{i}^{E}}{w_{i} h}+\frac{T_{i}^{E}-T_{i}^{N}}{w_{i} h}-\frac{B_{i}^{E}-B_{i}^{N}}{w_{i} h}
$$

où $S_{i}^{E}$ indique le montant des cotisations sociales en cas d'emploi ; $T_{i}^{E}$ et $T_{i}^{N}$ l'impôt direct en cas d'emploi et de nonemploi, respectivement ; et $B_{i}^{E}$ et $B_{i}^{N}$ les transferts sociaux en cas d'emploi et de non-emploi, respectivement.

\section{Taux marginal effectif: marge intensive}

Le taux marginal effectif $m_{i}$ mesure l'incidence du système, prélèvements et transferts confondus, suite à une augmentation marginale du revenu du travail. Ce taux donne une mesure du poids de l'incitant financier associé à une intensification de l'effort, soit une augmentation des heures de travail, soit la possibilité d'obtenir des primes ou l'accès à une promotion (Callan et al. [2011]). L'équation (8) indique la manière dont le taux marginal effectif est calculé :

$$
m_{i}=\frac{\partial T\left(w_{i} h\right)}{\partial w_{i} h}
$$


Plus concrètement, dans les micro-simulations présentées dans les sections suivantes, le taux $m_{i}$ individuel est calculé sur la base d'une augmentation hypothétique de $5 \%$ du revenu brut du travail $w_{i} h$.

\section{Coût marginal des fonds publics (MCPF)}

Comme indiqué plus haut, MCPF indique le coût en termes de bien-être résultant de la distorsion additionnelle créée par un instrument fiscal utilisé pour lever un euro additionnel. Ce coût marginal est en fait égal à $M C P F-1$ (Dahlby [2008]). En principe, nous devrions être capables de calculer ce taux marginal pour tous les individus de la population potentiellement disponibles sur le marché du travail. Comment peut-on agréger cette mesure d'efficience pour l'ensemble des individus?

Se servant d'un cadre d'analyse comportemental, Kleven et Kreiner [2006] assument que chaque unité de consommation est évaluée de la même manière à travers toute l'échelle de distribution de revenus ${ }^{11}$. Sur base de cette hypothèse, et en suivant Kleven et Kreiner, MCPF est calculée en partant de l'expression suivante :

$$
M C P F=\frac{1}{\sum_{i=1}^{10}\left[1-\frac{m_{i}}{1-m_{i}}\left(\phi_{i} \varepsilon_{i}^{c}-\theta_{i}\right)-\frac{\tau_{i}}{1-m_{i}} \eta_{i}\right] s_{i}}
$$

Dans le dénominateur de cette équation on trouve une somme pondérée des taux marginaux $m_{i}$ (extensif) et $\tau_{i}$ (intensif). Les variables de pondération sont, dans le premier cas, les deux composantes de l'élasticité marginale intensive, $\varepsilon_{i}=\varepsilon_{i}^{c}-\theta_{i}$, c'est-à-dire l'élasticité compensée $\varepsilon_{i}^{c}$, et l'effet revenu, notés $\theta_{i}$ et, dans le deuxième cas, l'élasticité de la marge extensive $\boldsymbol{\eta}_{i}$, conditionnée par le fait de participer au marché du travail. Deux autres termes interviennent dans ce calcul : $\phi_{i}$ est une mesure de la progressivité du changement marginal introduit dans le système de taxation (hors transferts), et $s_{i}$ indique dans quelle 
proportion la somme des taxes (hors transferts) payées par l'individu (ou le groupe) $i$ augmente, par rapport à l'accroissement observé pour l'ensemble de la population.

Afin de simplifier ce cas général, nous introduisons deux hypothèses dans le calcul du MCPF. D’une part, nous négligeons l'effet revenu dans la mesure d'élasticité correspondant à la marge intensive $\left(\theta_{i}=0\right)$ et, d'autre part, nous postulons $\phi_{i}=1 \mathrm{ou}$, en d'autres termes, MCPF est calculée sous l'hypothèse que le changement marginal n'affecte pas la progressivité de l'impôt (suite à un changement proportionnel de tous les taux marginaux du barème). L'équation (9) devient ainsi :

$$
M C P F=\frac{1}{\sum_{i=1}^{10}\left[1-\frac{m_{i}}{1-m_{i}} \varepsilon_{i}-\frac{\tau_{i}}{1-m_{i}} \eta_{i}\right] s_{i}}
$$

En avançant dans la suite de cet article, il faut remarquer que nous utiliserons des valeurs stylisées de $\varepsilon_{i}$ et de $\boldsymbol{\eta}_{i}$, les deux variables censées résumer le comportement des individus sur le marché du travail face aux incitants financiers. Ces valeurs, par décile de revenu brut de travail, sont celles utilisées auparavant par Kleven et Kreiner [2006], reproduites dans le tableau n ${ }^{\circ} 2$.

\section{Tableau 2}

\section{Elasticités de l'offre de travail}

\begin{tabular}{ccc}
\hline Déciles & $\begin{array}{c}\text { Marge } \\
\text { intensive } \\
\varepsilon_{i}\end{array}$ & $\begin{array}{c}\text { Marge } \\
\text { extensive } \\
\eta_{i}\end{array}$ \\
\hline $1-2$ & 0,1 & 0,4 \\
$3-4$ & 0,1 & 0,3 \\
$5-6$ & 0,1 & 0,2 \\
$7-8$ & 0,1 & 0,1 \\
$9-10$ & 0,1 & 0,0 \\
\hline
\end{tabular}

Source: Kleven et Kreiner [2006]. 


\section{Survol des réformes}

Notre objectif est de prendre en compte de manière conjointe les réformes touchant à l'impôt sur le revenu des personnes physiques (IPP), les cotisations sociales et les transferts de sécurité sociale au cours des deux dernières décennies. Sans vouloir être exhaustifs, nous passons en revue ici les principales réformes afin d'illustrer quelle a été leur orientation générale.

\section{Impôt sur le revenu des personnes physiques (IPP)}

Au cours de la période considérée, c'est en 2001 qu'a eu lieu la réforme la plus importante de l'IPP. En premier lieu, elle a aboli les taux marginaux les plus élevés $(52,5 \%$ et $55 \%$ ) et introduit un crédit d'impôt pour les personnes à faible revenu du travail. Ensuite elle a éliminé des différences dans la manière de taxer les revenus des ménages, isolés ou en couple : $i$ ) individualisation des crédits d'impôt pour les revenus de remplacement ; ii) abattements d'impôt identiques pour couples mariés et cohabitants légaux ; iii) taxation séparée des revenus hors travail; iv) extension du quotient familial aux cohabitants légaux. Enfin, une série d'autres mesures visaient une meilleure prise en compte du coût des enfants : $i$ ) crédit d'impôt pour enfant à charge pour certaines tranches de revenu ; ii) augmentation du revenu minimum afin de bénéficier du statut d'enfant à charge pour les ménages monoparentaux ; iii) extension du crédit d'impôt additionnel à tous les ménages monoparentaux (Cantillon et al. [2003]).

En dehors de cette réforme importante, l'IPP a subi des réformes mineures à d'autres moments :

- en 1992-1993 le traitement fiscal de l'épargne de long terme fut modifié de manière significative. D'une part, le taux de taxation est passé de $16,5 \%$ à $10 \%$ et, d'autre part, la déduction existante sur les assurances vie et les capitaux remboursés 
dans le cadre des prêts hypothécaires, fut remplacée par une réduction d'impôt ;

- en 1993 un supplément d'impôt de crise de 3 \% fut introduit. Puis supprimé en 2002 ;

- en 2005 c'est le traitement fiscal du revenu imputé de la maison propre (revenu cadastral) qui subit une modification importante. Jusqu'à cette année-là, ce revenu faisait partie du revenu soumis à l'impôt, bénéficiant dans certains cas de réductions. A partir de 2005 le revenu cadastral est devenu libre d'impôt et pour les crédits hypothécaires contractés à partir de 2005, les réductions d'impôt furent remplacées par le bonus logement (Verbist et Vanhille [2012]).

\section{Cotisations sociales}

Au cours des années 1990, la seule réforme importante a été l'introduction, en 1994, d'une cotisation sociale complémentaire calculée sur base du revenu imposable et du statut familial du travailleur.

A partir de 2000, les travailleurs moins qualifiés bénéficient d'une réduction de cotisations sociales personnelles, le bonus à l'emploi. Pour des rémunérations brutes mensuelles inférieures à un certain seuil minimum, $1372 €$ (à prix de 2009), les cotisations personnelles sont remboursées forfaitairement, $105 €$ (prix de 2009), ce qui représente près de deux-tiers du total. Au-delà de ce seuil minimum, le montant du remboursement diminue, jusqu'à disparaître pour des salaires égaux ou supérieurs au seuil maximum, $1634 €$ (à prix de 2009).

Par la suite, au cours de la période 2001 à 2007, le bonus à l'emploi subit deux réformes importantes. D'une part, pour les salaires inférieurs au seuil minimum, le remboursement correspond pratiquement à l'ensemble des cotisations sociales personnelles. D'autre part, le seuil maximum pour bénéficier du bonus à l'emploi augmente de près de $50 \%$ en termes réels, $2199 €$ (à prix de 2009). 


\section{Prestations de sécurité sociale}

Nous passons en revue ici les principales réformes en matière de transferts sociaux correspondant aux différentes branches de la sécurité sociale et de l'assistance sociale.

\section{Allocations familiales}

De manière générale, le système des allocations familiales n'a pas subi des grands changements au cours de la période que nous considérons. Une réduction est intervenue en 1997 dans le supplément pour âge accordé aux enfants de premier rang et deux suppléments d'allocations furent introduits en 2007 : i) une prime de retour à l'école pour des enfants âgés de moins de 25 ans et, ii) des allocations complémentaires pour des familles monoparentales.

\section{Maladie-invalidité}

Jusqu'en 2006, une seule distinction était faite entre les allocations au taux "isolé " et au taux "ménage ». A partir de 2007 la catégorie " cohabitant " fut introduite en suivant le même critère appliqué par l'assurance chômage. Le taux de remplacement pour les bénéficiaires à taux "isolé " est passé graduellement de $45 \%$ en 1988 à $50 \%$ en 2003, $53 \%$ en 2008 et $55 \%$ en 2010.

\section{Pensions}

En Belgique, en dehors du régime de pensions des fonctionnaires qui relève directement du budget de l'Etat, trois autres régimes relèvent de la Sécurité sociale : travailleurs salariés, travailleurs indépendants et revenu garanti. Nous nous intéressons ici au régime de Sécurité sociale, dont dépendent une grande majorité des pensionnés, en nous limitant uniquement à la situation des bénéficiaires et aux réformes qui les concernent. Le montant des pensions, comme les autres transferts sociaux, est régulièrement adapté en suivant l'évolution de l'indice des prix, néanmoins, depuis 2002, les pensions de retraite bénéficient 
également de l'adaptation au bien-être. Cette adaptation a pour objet d'éviter une trop forte détérioration du montant de la pension, notamment par rapport à l'évolution des salaires dans l'économie. L'annexe $\mathrm{n}^{\circ} 2$ présente les changements intervenus depuis 2002. Comme on peut le constater, ces augmentations interviennent de manière assez régulière et concernent généralement les cohortes de pensionnés ayant pris leur retraite des années auparavant.

\section{Allocations de chômage}

Au fil du temps il y a eu une convergence des taux de remplacement. Pour les chômeurs avec partenaire à charge, le taux de remplacement "ménage " est resté fixé à $60 \%$ tout au long de la période. En revanche, pour d'autres catégories de chômeurs il y a eu, entre 1988 et 2012, des augmentations significatives : de $40 \%$ à $55 \%$ et de $35 \%$ à $40 \%$ pour les chômeurs de longue durée (plus de 12 mois de chômage) au taux " isolé " et " cohabitant ", respectivement; et de $55 \%$ à $60 \%$ pour les chômeurs de courte durée au taux " cohabitant " ${ }^{12}$. Il faut aussi noter qu'au cours du temps les montants minimum et maximum des allocations de chômage, autres paramètres du système, ont augmenté plus rapidement que le salaire moyen, en particulier pour les prestations aux taux " isolé » et " cohabitant ».

\section{Assistance sociale}

En l'année 2000, les régimes existants ont connu des changements importants, aussi bien au niveau des allocations de revenu minimum d'assistance (Minimex) que des allocations de revenu minimum garanti pour les personnes âgées (Grapa). En plus d'une augmentation significative des allocations, le gouvernement a simultanément introduit l'individualisation des droits à la garantie de revenus. A titre d'exemple, pour un couple de bénéficiaires, le montant auquel chacun des partenaires a droit est égal à la moitié du montant dont le couple aurait bénéficié en l'absence de réforme. 


\section{Estimation et résultats}

Nos estimations sont basées sur les données récoltées dans le cadre de l'enquête EU-SILC (European Union Statistics on Income and Living Conditions) conduite par Eurostat, l'office des statistiques de l'Union européenne. Cette enquête annuelle auprès des ménages est la référence pour l'étude des inégalités et de la pauvreté en Europe. Elle contient des informations très détaillées sur les caractéristiques démographiques et socioéconomiques des ménages ainsi que sur les revenus dont ils disposent. Dans le cadre de cette étude nous utilisons exclusivement les données EU-SILC 2010, lesquelles portent sur les revenus de 6100 ménages belges en $2009^{13}$.

Figure 1

Structure socio-économique de la population par décile de revenu

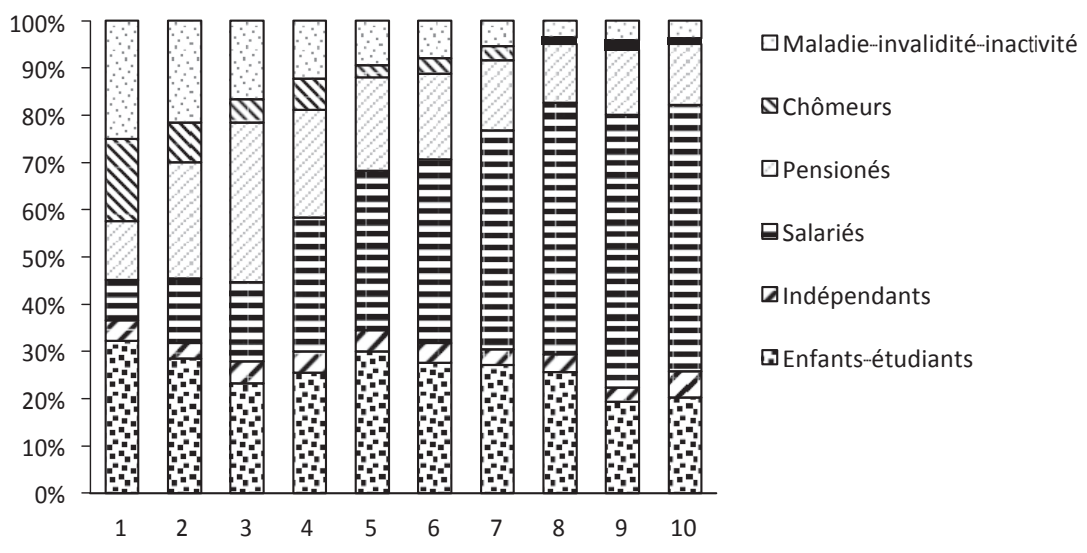

Source : EU-SILC [2010]. Déciles : revenu disponible du ménage par unité de consommation (échelle OCDE).

En fonction de différentes analyses, les contours de la population étudiée vont varier. Ainsi, en premier lieu, l'effet des réformes sur le pouvoir d'achat sera analysé pour l'ensemble de la population en observant les changements intervenus dans le 
revenu disponible du ménage. Tous les individus faisant partie de la population seront groupés par décile de revenu, celui-ci étant défini comme le revenu du ménage par unité de consommation (revenu standardisé). La figure $\mathrm{n}^{\circ} 1$ présente la structure socioéconomique de la population belge en 2010 à partir d'EU-SILC.

En deuxième lieu, afin de mesurer l'impact des réformes sur l'inégalité de revenus (l'équité) et l'efficacité, on s'intéressera au sousensemble constitué par la population en âge de travailler, que nous définissons comme étant la population âgée entre 18 et 59 ans $^{14}$.

Le tableau $\mathrm{n}^{\circ} 3$ indique comment la taille de l'échantillon, et sa représentativité de l'ensemble de la population, varient en fonction des mesures.

\section{Tableau 3}

\section{Taille et représentativité des échantillons par type de mesure}

\begin{tabular}{llcc}
\hline Mesures & Population & EU-SILC 2010 & $\begin{array}{c}\text { Belgique 2010 } \\
\text { (x1000) }\end{array}$ \\
\hline Revenu disponible & Ensemble & 14.700 & 10.682 \\
Equité et efficacité & $\begin{array}{l}\text { En âge de travailler } \\
(18-59 \text { ans) }\end{array}$ & 7.439 & 5.561 \\
\hline
\end{tabular}

Source : EU-SILC 2010.

\section{Effets redistributifs}

La figure $\mathrm{n}^{\circ} 2$ montre les taux de variation du revenu disponible par décile de revenu, par application du système fiscal en vigueur en 2012, comparé au système en vigueur en 1992. Les résultats, exprimés en points de pourcentage du revenu disponible en 1992, indiquent que le système fiscal est devenu plus généreux. En d'autres termes, étant donné le revenu du ménage avant taxes et transferts sociaux et étant données les caractéristiques socio-économiques de l'individu et du ménage dont il fait partie, son revenu disponible, après taxes et transferts sociaux, est supérieur sous le système en vigueur en 2012 que sous celui en vigueur en 1992. En moyenne, ceteris paribus, son revenu disponible serait inférieur de 2,9\% en 1992. 
En regardant plus en détail les déciles de revenu, on observe néanmoins des variations importantes : $12,8 \%$ d'augmentation dans le décile inférieur contre $1,2 \%$ dans le décile supérieur ${ }^{15}$. Sur la figure $\mathrm{n}^{\circ} 2$ on peut aussi distinguer les effets des réformes pour chacun des différents éléments du système taxes/bénéfices. Clairement, les ménages situés dans les premiers déciles de revenus sont ceux qui ont bénéficié proportionnellement le plus de l'amélioration des transferts sociaux et des déductions d'impôts, tandis que pour les ménages dans les déciles supérieurs ces améliorations ont été compensées par des augmentations des cotisations sociales.

Figure 2

\section{Taux de variation dans le revenu disponible (1992-2012)}

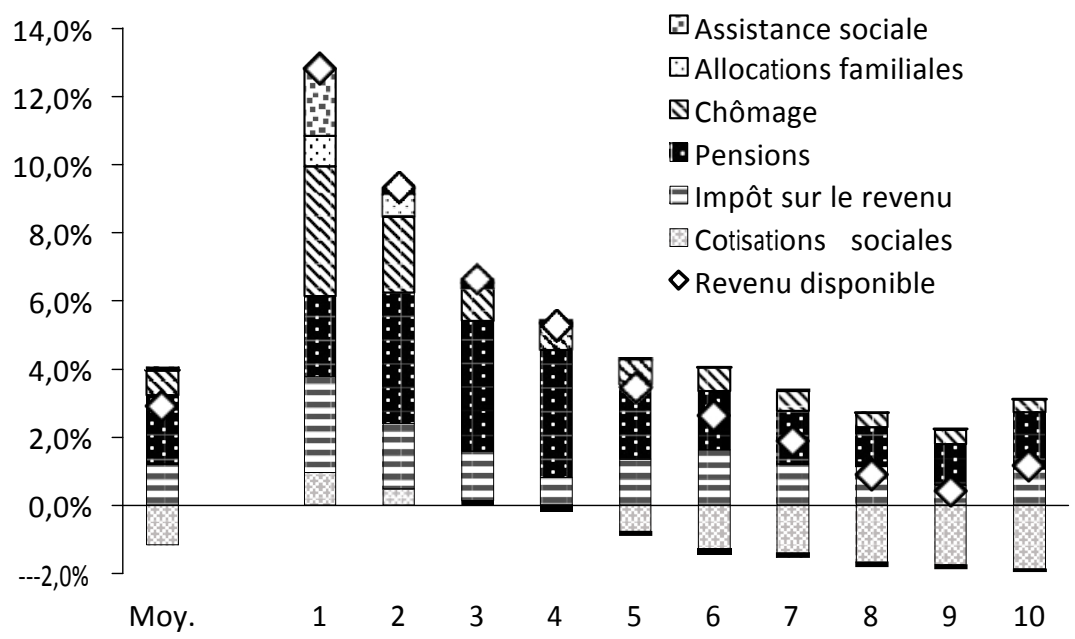

Source : EU-SILC 2010 et calculs propres avec Mefisto-Euromod. Déciles du revenu disponible du ménage par unité de consommation.

Dans les sections suivantes nous passons en revue les effets redistributifs des réformes pour trois sous-périodes : 19922001, 2001-2007 et 2007-2012.

Evolution de 1992 à 2001

Les deux premiers gouvernements de cette période, 1992-1995 (Dehaene I) et 1995-1999 (Dehaene II), ont suivi des politiques 
d'austérité en vue de respecter les critères de Maastricht et rejoindre l'euro $^{16}$. Le taux des cotisations sociales et l'impôt sur le revenu ont été augmentés de manière proportionnelle. C'est le gouvernement suivant, Verhofstadt I (1999-2003), qui introduit en 2000 la réduction de cotisations sociales personnelles pour les travailleurs moins qualifiés, le bonus à l'emploi. Nos estimations pour l'ensemble de la période 1992-2001 sont présentées sur la figure $\mathrm{n}^{\circ} 3$. Elles montrent que le revenu disponible aurait diminué de 2,3\% en moyenne, diminution due essentiellement à l'augmentation des cotisations sociales et de l'impôt sur le revenu.

Figure 3

\section{Taux de variation dans le revenu disponible (1992-2001)}

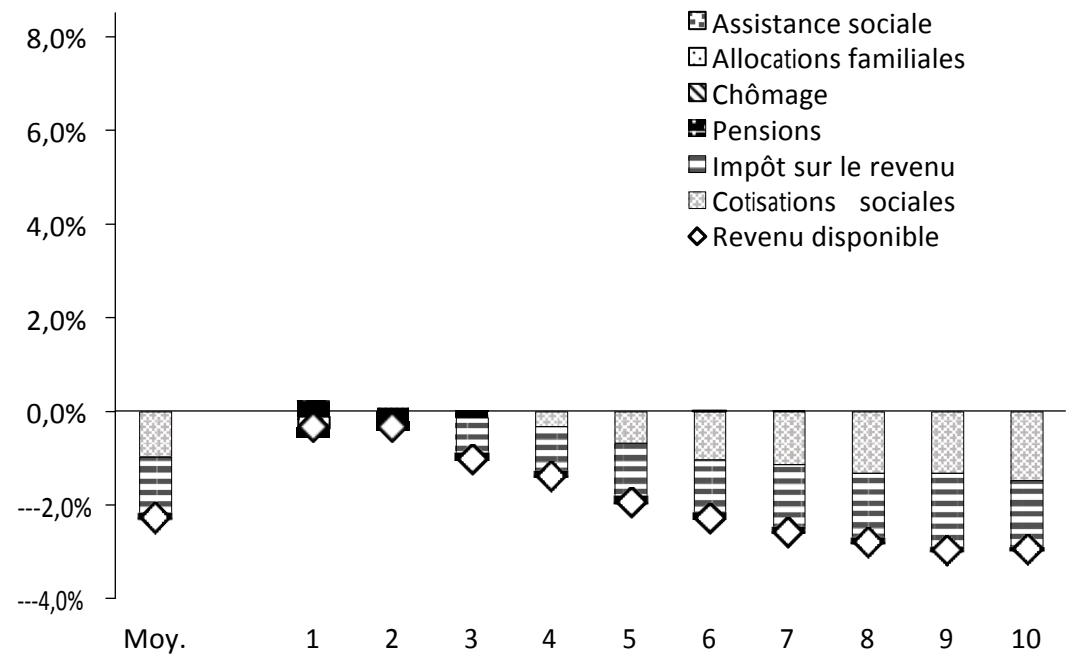

Source : EU-SILC 2010 et calculs propres avec Mefisto-Euromod. Déciles du revenu disponible du ménage par unité de consommation.

Evolution de 2001 à 2007

Cette période couvre les changements les plus importants introduits par les gouvernements Verhofstadt I (1999-2003) et II (2003-2007). Au cours de cette période, le revenu disponible des ménages a augmenté en moyenne de 4,4 \% grâce à des réformes favorables du système fiscal (voir figure $\mathrm{n}^{\circ} 4$ ). L'augmentation a 
été proportionnellement plus élevée pour les ménages dans le premier décile $(8,3 \%)$, mais également pour les ménages dans l'ensemble de la distribution de revenus (3,5\% à 5,8 \%). C'est surtout la réforme de l'impôt sur le revenu (réforme Reynders) qui a été à la base de de cette augmentation. Elle a consisté dans la diminution du taux marginal d'imposition et dans l'introduction d'un nombre important de déductions fiscales. Au même moment le bonus à l'emploi a été étendu de manière à donner droit à une diminution plus importante de cotisations sociales. C'est également au cours de la même période, à partir de 2002, que des adaptations régulières des prestations sociales - assistance sociale, pensions et allocation de chômage - au bien-être ont été introduites. Enfin, les familles à faibles revenus ont pu bénéficier de compléments d'allocations familiales alors que simultanément, et d'une manière générale, les allocations familiales perdaient de la valeur en termes réels, n'étant pas indexées complétement à l'évolution des prix à la consommation.

Figure 4

\section{Taux de variation dans le revenu disponible (2001-2007)}

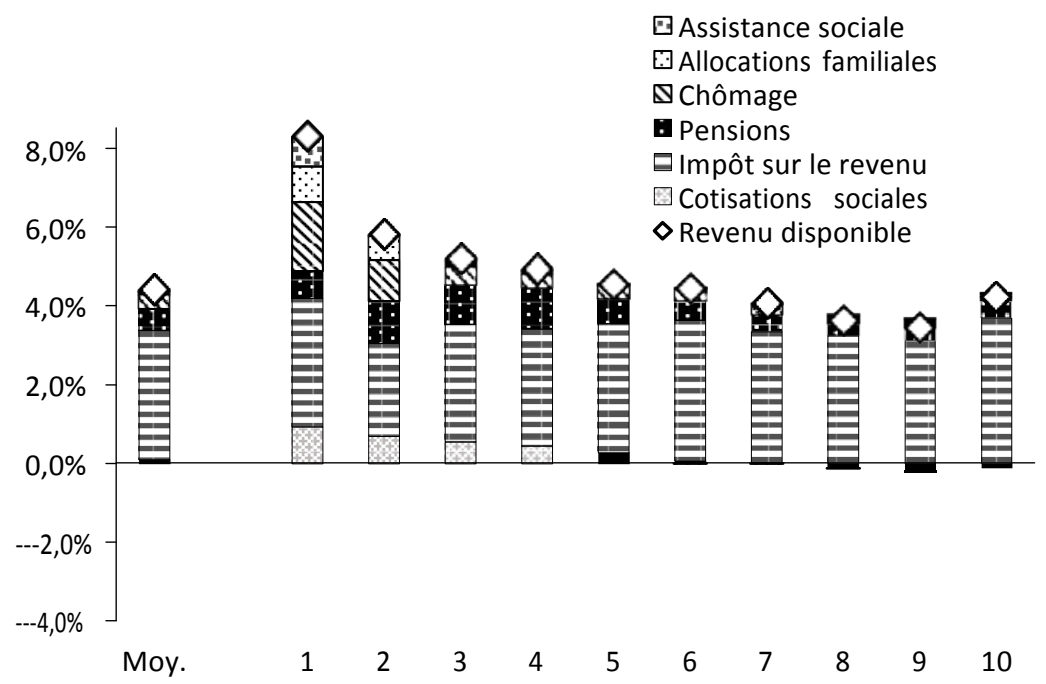

Source : EU-SILC 2010 et calculs propres avec Mefisto-Euromod. Déciles du revenu disponible du ménage par unité de consommation. 
Evolution de 2007 à 2012

Nos estimations pour cette période, présentées sur la figure $\mathrm{n}^{\circ} 5$, indiquent que pendant et après la crise financière, les réformes du système fiscal ont eu comme conséquence une augmentation du revenu disponible d'approximativement $0,9 \%$ en moyenne. Cet effet est surtout le résultat de l'adaptation au bien-être des allocations de revenu minimum garanti (assistance sociale), des allocations de chômage et des pensions. L'abrogation de certains avantages fiscaux a eu comme effet une diminution du revenu disponible des ménages, de l'ordre $0,8 \%$. En somme, les réformes introduites bénéficient aux ménages les plus pauvres, $4,5 \%$ dans le revenu disponible au sein du premier décile, tandis que dans les déciles supérieurs, l'effet a été proche de zéro.

\section{Figure 5}

\section{Taux de variation dans le revenu disponible (2007-2012)}

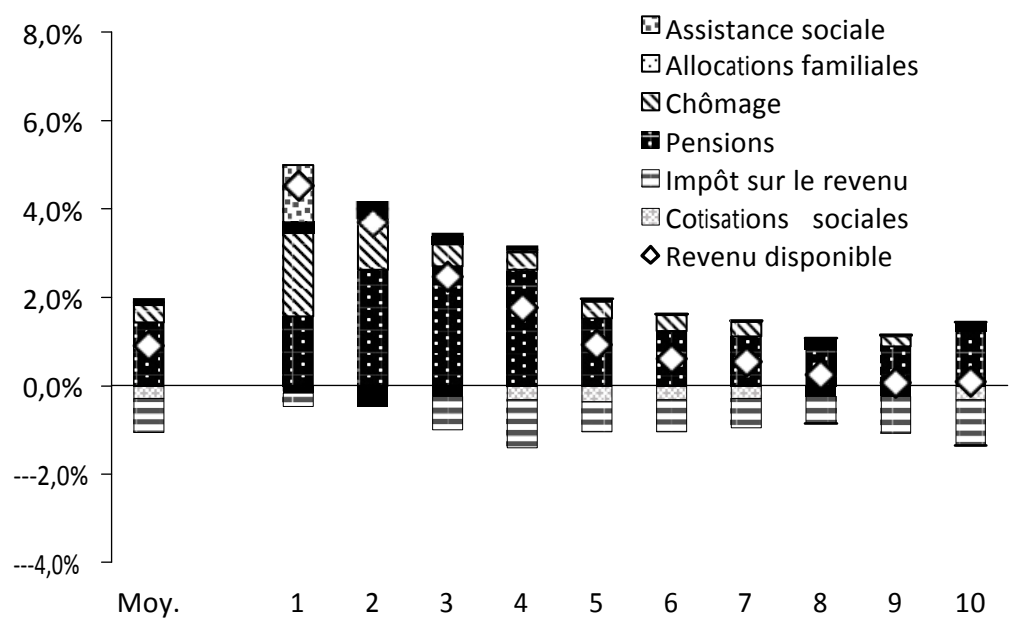

Source : EU-SILC 2010 et calculs propres avec Mefisto-Euromod. Déciles du revenu disponible du ménage par unité de consommation.

\section{Equité}

Comme on vient de le constater, le système fiscal belge a joué un rôle redistributif positif au cours de la période. L'évolution 
de l'indice de Reynolds-Smolensky, présentée dans le tableau $\mathrm{n}^{\circ} 4$, résume bien cette situation. On observe, en effet, une augmentation de 9,9 en 1992 à 11,4 en 2012, ce qui correspond à une augmentation de l'effet redistributif des réformes, prises dans leur ensemble. Cette amélioration est par ailleurs assez régulière au cours des trois sous-périodes, néanmoins quand on s'intéresse à la décomposition de l'indice on constate un changement important intervenu après l'année 2001. On observe une forte augmentation dans la progressivité du système, l'indice de Kakwani passant de 26,9 en 2001 à 32,3 en 2007 (puis à 33,9 en 2012). Sur la même période, le taux moyen de taxation (taxes/bénéfices) tombe de 32,3\% à 29,4\%. Ce sont les réformes introduites après l'année 2000, en particulier l'importante réforme de l'impôt direct (impôt des personnes physiques) entre 2001 et 2003, le bonus à l'emploi et les adaptations au bien-être opérées sur les allocations de chômage et le revenu minimum garanti, qui sont à la base de ces effets ${ }^{17}$.

\section{Table 4}

\section{Effet redistributif, progressivité et taux de taxation moyen}

\begin{tabular}{lcccc}
\hline & 1992 & 2001 & 2007 & 2012 \\
\hline $\begin{array}{l}\text { Effet redistributif } \\
\text { (Indice de Reynolds-Smolensky) }\end{array}$ & 9,9 & 10,4 & 11,0 & 11,4 \\
$\begin{array}{l}\text { Progressivité } \\
\text { (Indice de Kakwani) }\end{array}$ & 27,4 & 26,9 & 32,3 & 33,9 \\
Taux de taxation moyen & 30,9 & 32,3 & 29,4 & 29,3 \\
\hline
\end{tabular}

\section{Incitations sur la participation au marché de travail}

\section{Marge extensive}

Sur la figure $\mathrm{n}^{\circ} 6$, on observe l'évolution du taux marginal de taxation sur la participation au marché du travail, marge extensive ou PTR, pour " participation taxe rate " : 75,7 \% en 1992 ; $74,8 \%$ en $2001 ; 73,9 \%$ en 2007 et $77,2 \%$ en 2012 . Alors que le niveau implicite d'imposition sur la participation au marché de travail était très élevé, en particulier pour les premiers déciles 
(définis en fonction du revenu du travail), il diminue jusqu'en 2007, puis augmente à la fin de la période pour l'ensemble des déciles. Ce résultat est en rapport direct avec l'effet des réformes présentées plus loin, sur le niveau des allocations de chômage, des contributions sociales et de l'impôt sur le revenu.

Figure 6

Taux marginal extensif (PTR), par décile de revenu (\%)
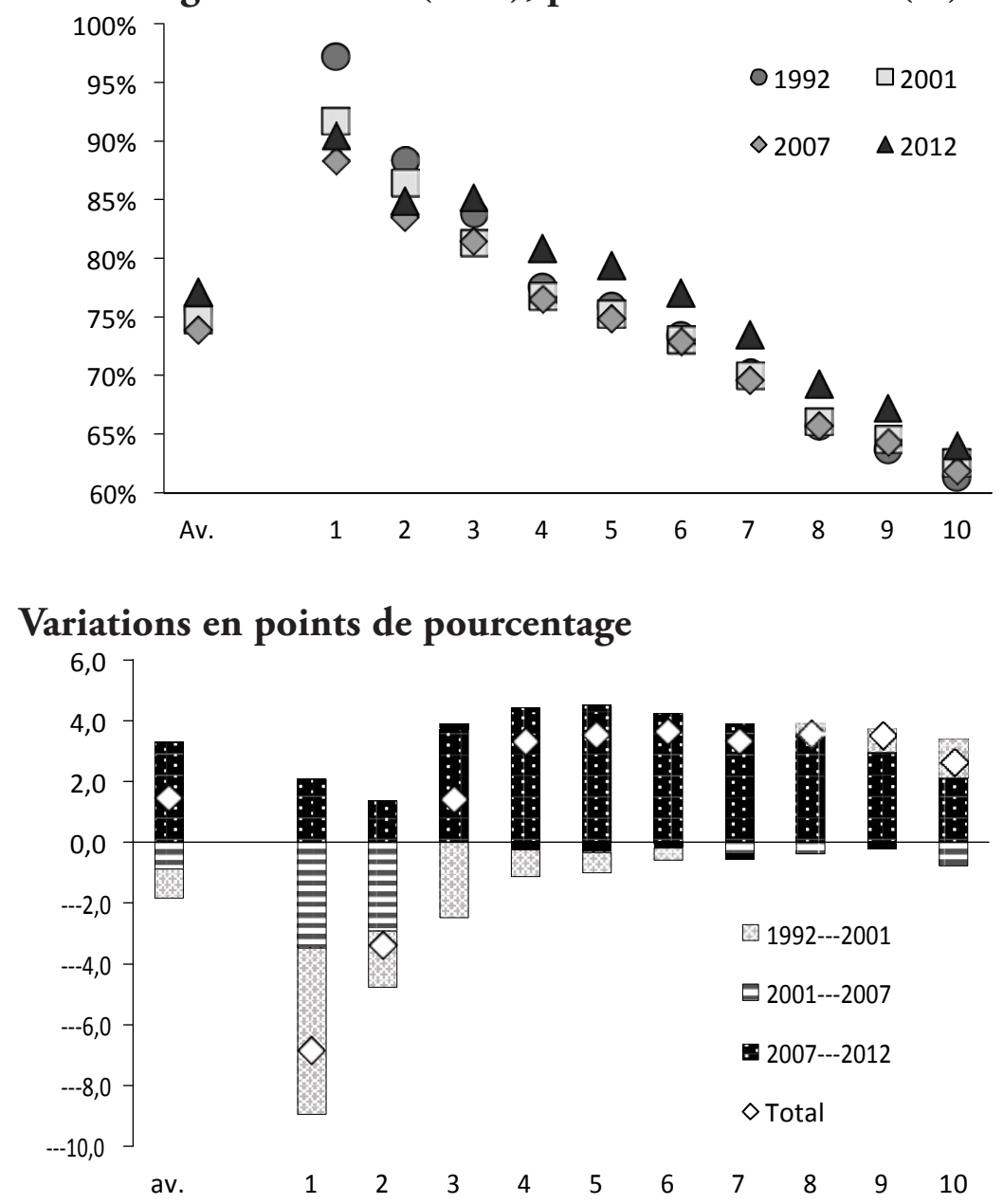

Source : SILC 2010 ; calculs propres en utilisant Mefisto-Euromod ; déciles de revenu du travail. 
Tout d'abord, si au cours des années 1990, l'augmentation proportionnelle des contributions de sécurité sociale (voir figure $\mathrm{n}^{\circ} 3$ ) a donné lieu à une augmentation légère des PTR pour les trois déciles supérieurs de la distribution, elle a également eu comme conséquence une diminution des PTR pour l'ensemble des premiers déciles, en particulier le premier. Ceci s'explique par l'introduction du bonus à l'emploi en 2000.

Ensuite, pendant la période 2001-2007, c'est à nouveau le bonus à l'emploi, en fait son extension à des catégories de revenus plus élevés, qui a donné lieu à une diminution importante de la marge extensive pour les deux déciles inférieurs, c'est-à-dire les revenus les plus faibles.

L’objectif de cette réforme était justement de rendre l'emploi financièrement plus attractif pour les travailleurs moins qualifiés via la réduction des contributions sociales et le relèvement du revenu minimum imposable. Depuis l'introduction de cette réforme, le taux PTR aurait diminué de $6,8 \%$ pour le premier décile et de 3,4\% pour le second. Ceci confirme l'orientation de cette mesure, ciblée en faveur des salaires les plus bas.

Enfin, au cours de la période 2007-2012, on assiste à l'augmentation des PTR pour l'ensemble de l'échelle des salaires. Cet effet est pratiquement entièrement expliqué par l'augmentation du montant des allocations de chômage ${ }^{18}$. En effet, l'augmentation du revenu hors travail implique un incitant financier puissant en défaveur de l'emploi. Nous avons estimé cet effet entre 7,0 et 8,6 \%, en termes de PTR, entre 1992 et 2012 pour les cinq premiers déciles. Dans la mesure où les travailleurs dans les déciles de salaires supérieurs bénéficient proportionnellement moins de ces augmentations (adaptations au bien-être des allocations sociales) du fait de leur plafonnement, l'augmentation des PTR a été plus limitée pour ces catégories, ce qui donne, en moyenne, 4,4\% d'augmentation pour l'ensemble de la population en âge de travailler.

\section{Marge intensive}

Sur la figure $\mathrm{n}^{\circ} 7$ on remarquera tout d'abord la forme générale en $U$ du taux marginal effectif de taxation (METR), avec des 
taux plus élevés pour les premiers et les derniers déciles. Cette forme est en accord avec les prescriptions de la littérature sur la taxation optimale des revenus de travail (voir Saez [2002]) ${ }^{19}$.

Nous remarquerons ensuite qu'en moyenne le taux marginal effectif de taxation (METR) a augmenté de 52,2\% à 55,3\% au cours de la période 1992 à 2012. Néanmoins, ces valeurs moyennes cachent d'importantes variations au niveau des déciles. En effet, comme on peut le constater sur la deuxième partie de la figure $\mathrm{n}^{\circ} 7$, c'est surtout au sein des premiers déciles que les changements les plus importants ont eu lieu. Ils sont en rapport avec les réformes introduites au cours des trois sous-périodes analysées.

Premièrement, l'introduction du supplément de l'impôt de crise et de la cotisation supplémentaire de sécurité sociale au début des années 1990 a eu comme effet l'augmentation du METR de manière substantielle pour le premier décile des salaires, plus de $10 \%$, mais également pour toutes les catégories de travailleurs, malgré l'introduction en 2000 du bonus à l'emploi.

La période 2001-2007, est caractérisée par l'extension $\mathrm{du}$ bonus à l'emploi. Cette mesure, favorable aux plus bas salaires, s'éteint graduellement à mesure que le salaire augmente, ce qui se traduit par des taux marginaux effectifs plus élevés pour les deuxième et troisième déciles. En revanche, pour le premier décile c'est le traitement fiscal du bonus qui renforce davantage l'effet de cette réforme sur l'offre d'emploi. En effet, pour les travailleurs moins qualifiés, les deux effets, taux effectif (METR) et marge extensive (PTR), favorisent l'emploi. Ensuite on remarquera que la réforme de l'impôt sur le revenu des personnes physiques, intervenue en 2001-2003, celle qui a consisté en la diminution des taux marginaux les plus élevés ainsi que la suppression du complément d'impôt de crise, a eu très peu d'effet sur le taux marginal de taxation, en marge effective. On observe une diminution du METR pour les plus hauts niveaux de salaires, mais très faible, au cours de la période 2001-2007.

Enfin, au cours de la dernière période, les réformes ont essentiellement affecté le taux marginal intensif sur les premiers déciles. Rappelons-le, les réformes au cours de cette période ont surtout consisté dans l'augmentation des allocations sociales 
et ont eu comme première conséquence l'augmentation du taux marginal extensif (PTR). On observe une augmentation du METR pour le premier décile et une diminution pour les déciles suivants, en particulier le deuxième.

\section{Figure 7}

Taux marginal intensif (METR) par décile de revenu (\%)

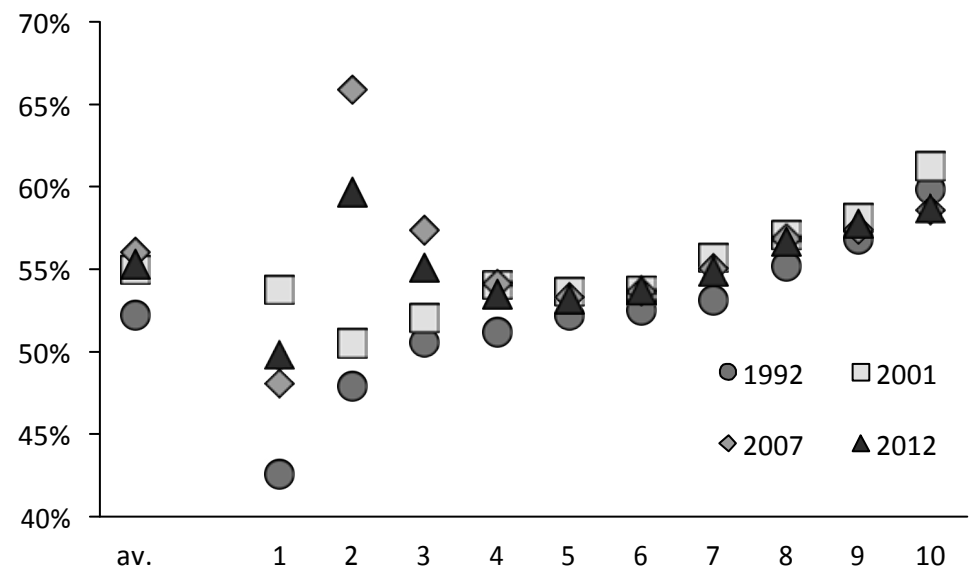

\section{Variations en points de pourcentage}

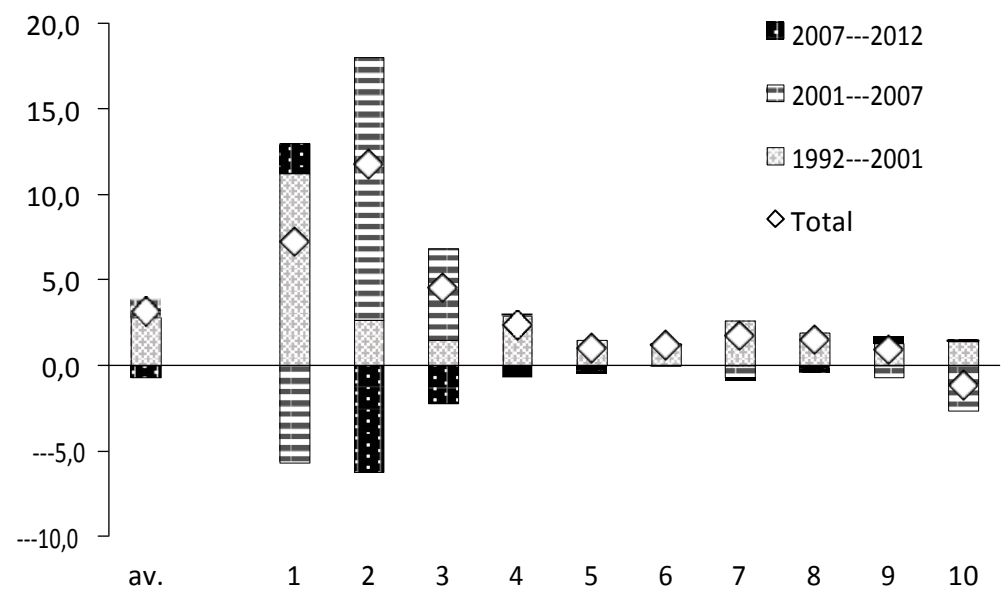

Source : SILC 2010 ; calculs propres en utilisant Mefisto-Euromod ; déciles de revenu du travail. 


\section{Le coût marginal des fonds publics (MCPF)}

C'est en nous servant des taux marginaux calculés dans les sections précédentes et des élasticités stylisées présentées dans le tableau $n^{\circ} 2$, que nous avons estimé le MCPF pour l'ensemble de la période. Nos résultats montrent que sa valeur a augmenté mais pas de manière constante. Pour l'année 1992 la valeur de MCPF était égale à 1,408 , ce qui signifie qu'une augmentation d'un euro du niveau des taxes (nettes des bénéfices et transferts sociaux) implique un coût de 40,8 centimes en termes de bienêtre. Entre 1992 et 2001 le MCPF augmente à 1,444. Ensuite, par l'effet des différentes réformes introduites au cours de la période 2001-2007, il reste inchangé et vaut 1,444 en 2007. Enfin, de 2007 à 2012 le MCPF s'accroît une nouvelle fois, jusqu'à 1,455. A la lecture de ces résultats, le système fiscal belge serait donc devenu, selon cette mesure, moins efficient au cours de deux dernières décennies.

\section{Equité et efficacité}

Comme nous l'avons fait remarquer dans l'introduction, l'objectif de cet article n'est pas de décrire un arbitrage potentiel entre équité et efficacité auquel les responsables auraient fait face au moment du choix des réformes. Les deux mesures agrégées que nous avons calculées, d'équité et d'efficacité, ne sont en fait utilisées ici que comme outils descriptifs. Comme elles ont été calculées sur base d'un même système fiscal, il n'y a pas de lien direct entre les effets redistributifs et les variations observées dans la mesure d'efficacité. Cette dernière est en effet une mesure agrégée du changement marginal hypothétique de l'offre de travail introduite ici pour traduire l'effet potentiel des réformes au travers des incitations agissant sur les comportements individuels sur le marché du travail.

La figure $\mathrm{n}^{\circ} 8$ met en perspective les résultats obtenus sur l'effet des réformes du système fiscal belge sur l'équité et l'efficacité au cours de la période 1992-2012. Nous avons reporté 
sur cette figure l'évolution de l'équité, l'indice de ReynoldsSmolensky, et de l'efficacité, mesurée comme $1-M C P F$. Le résultat des réformes indique un déplacement positif sur le plan de l'équité au fil du temps, simultanément à un déplacement négatif sur le plan de l'efficacité. En somme, des allocations sociales plus généreuses combinées à des abattements de taxes et de contributions sociales favorisant l'emploi mènent vers un système fiscal plus équitable. Cependant, l'augmentation des taux marginaux de taxation sur l'offre de travail réduit partiellement cet effet. Les distorsions plus importantes que l'on observe dans l'efficacité du système sont dues à l'augmentation des taxes et des cotisations sociales au cours de la première sous-période et au niveau plus élevé des prestations en cas de non-travail au cours de la dernière sous-période.

Figure 8

\section{Evolution des mesures d'équité et d'efficacité}

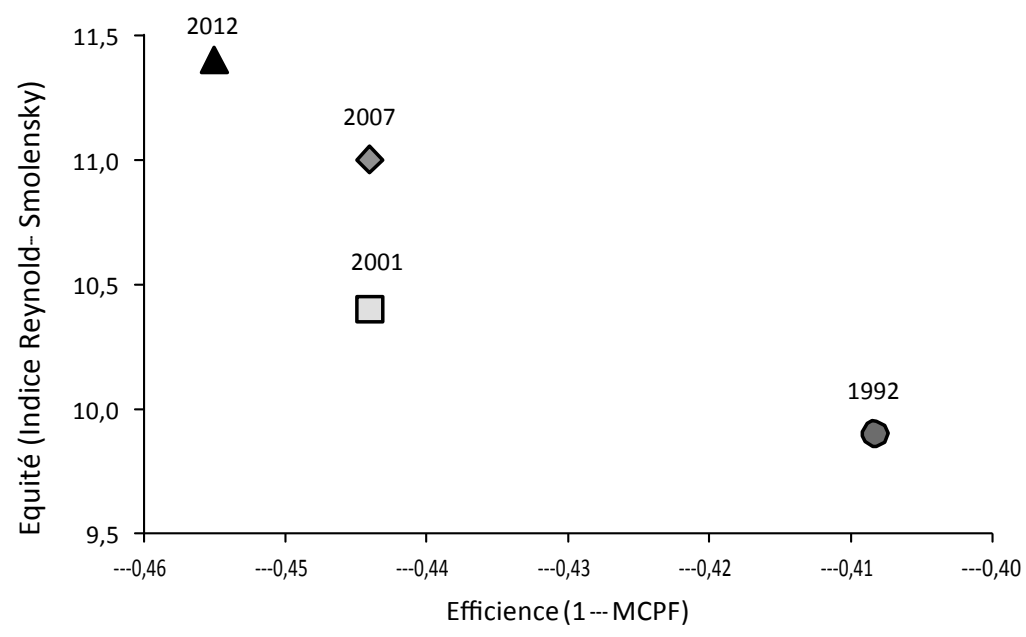

On constate sur la figure ${ }^{\circ} 8$ qu'équité et efficacité n'ont pas été nécessairement des objectifs opposés des réformes, comme le montre l'évolution pendant la période allant de 2001 à 2007. En d'autres mots, il est possible de faire évoluer 
positivement l'équité avec des mesures qui ont comme conséquence d'augmenter les revenus des ménages dans les premiers déciles plus rapidement que les hauts revenus (c'est ce qui s'est passé pendant la période 2001-2007), et en même temps de maintenir inchangé le coût du système en termes du bien-être. Les abattements de taxes et de cotisations sociales en faveur de l'emploi, le bonus à l'emploi, ont permis de réduire les distorsions sur le marché du travail, à la fois sur la marge extensive et intensive. Néanmoins, sur la même période, l'augmentation des allocations de chômage a eu comme conséquence une réduction substantielle de ces gains d'efficacité en ce qui concerne la participation au marché du travail (marge extensive).

Dans cet article nous avons montré qu'au cours des vingt dernières années, les réformes que le système fiscal belge a connues ont eu des effets positifs importants sur le revenu disponible des ménages. Sans ces réformes, les revenus moyens de l'ensemble des catégories (déciles) de revenu auraient été inférieurs.

Une autre conclusion de cette étude est que les réformes ont privilégié l'objectif redistributif. Les augmentations de taxes au cours des années 1990 ont épargné les catégories plus pauvres, lesquelles ont par ailleurs bénéficié de réductions d'impôts et de cotisations sociales. D'autre part, les allocations de chômage et les pensions ont subi une série d'adaptations au bien-être, en particulier pendant la période 2001-2007, qui ont surtout bénéficié aux ménages pauvres. Ces adaptations ont été poursuivies pendant la période 2007-2012 mais uniquement au bénéfice des pensionnés.

Enfin, contrairement aux effets positifs des réformes sur le plan de l'équité, les résultats en termes d'efficacité ont été plus mitigés et, de manière générale, moins favorables. En effet, le taux marginal d'imposition, en particulier celui pesant sur la participation au marché du travail (marge extensive) mais également celui sur 
le nombre d'heures (marge intensive), ont augmenté. La diminution importante des cotisations de sécurité sociale pour les travailleurs à revenu faible, bonus à l'emploi, a eu un effet favorable sur le taux implicite de taxation affectant la participation au marché du travail (PTR). C'est en encourageant la participation au marché du travail des moins qualifiés que les réformes ont eu un effet favorable sur l'efficacité. Inévitablement, l'augmentation des allocations de chômage, intervenue également au cours de la période, a eu l'effet inverse.

Il faut cependant rappeler que les résultats des microsimulations présentés ici reposent sur deux hypothèses importantes. D'une part, faute d'informations rétrospectives complètes, nous avons procédé aux simulations en considérant une structure sociodémographique inchangée et, d'autre part, afin de procéder à l'agrégation des taux marginaux d'imposition, nous nous sommes servi des élasticités d'offre de travail estimées ailleurs dans la littérature. Nous ne pensons pas que les conclusions de cette étude devraient différer substantiellement si nous disposions d'informations plus appropriées. Néanmoins, nous invitons le lecteur à la prudence dans l'interprétation des résultats.

Les auteurs remercient Bea Cantillon, Bernard Jurion, Frank Vandenbroucke et Guy Van Camp pour leurs commentaires et suggestions sur une version préliminaire de cet article. Ils sont également reconnaissants du soutien apporté à cette étude par la région flamande dans le cadre du projet "FLEMOSI:A tool for ex ante evaluation of socio-economic policies in Flanders " financé par l'IWT (Agency for Innovation by Science and Technology).

Les simulations présentées dans cette étude sont basées sur les données EU-SILC 2010 pour la Belgique et ont été réalisées à l'aide de la version F6.36+ d'Euromod. Les auteurs assument l'entière responsabilité des résultats et conclusions présentés ici.

André Decoster est Full Professor au Center for Economic Studies, KU Leuven.

Adresse: Faculty of Business and Economics, Naamsestraat, 69, B-3000 Leuven, Belgique. Email : andre.decoster@econ.kuleuven.be

Sergio Perelman est Chargé de cours honoraire à HEC-Ecole de Gestion, Université de Liège. Adresse : Bd.du Rectorat 7 (B31),B-4000Liège,Belgique.Email: sergio.perelman@ulg.ac.be 
120 A. Decoster, S. Perelman, D. Vandelannoote, T. Vanheukelom et G. Verbist

Dieter Vandelannoote est PhD Student à Herman Deleeck Center for Social Policy, University Antwerp.

Adresse : Sint-Jacobstraat 2, B-2000 Antwerpen, Belgique.

Email : dieter.vandelannoote@uantwerpen.be

Toon Vanheukelom est PhD Student au Center for Economic Studies, KU Leuven.

Adresse: Faculty of Business and Economics, Naamsestraat, 69, B-3000 Leuven, Belgique.

Email:Toon.Vanheukelom@kuleuven.be

Gerlinde Verbist est Senior Researcher à Herman Deleeck Center for Social Policy, University Antwerp.

Adresse : Sint-Jacobstraat 2, B-2000 Antwerpen, Belgique.

Email: gerlinde.verbist@uantwerpen.be 
A. Decoster, S. Perelman, D. Vandelannoote, T. Vanheukelom et G. Verbist 121

\section{Annexe 1}

\section{Illustration du calcul du revenu disponible contrefactuel}

Dans cette annexe nous présentons une illustration de la façon dont nous procédons pour le calcul du revenu net disponible contrefactuel en 2001 à partir de la situation observée en 2009. L'exemple choisi est celui de l'impôt sur le revenu des personnes physiques (IPP), plus précisément l'effet résultant de la nonindexation des tranches d'imposition.

Figure 9

\section{Calcul du revenu contrefactuel}

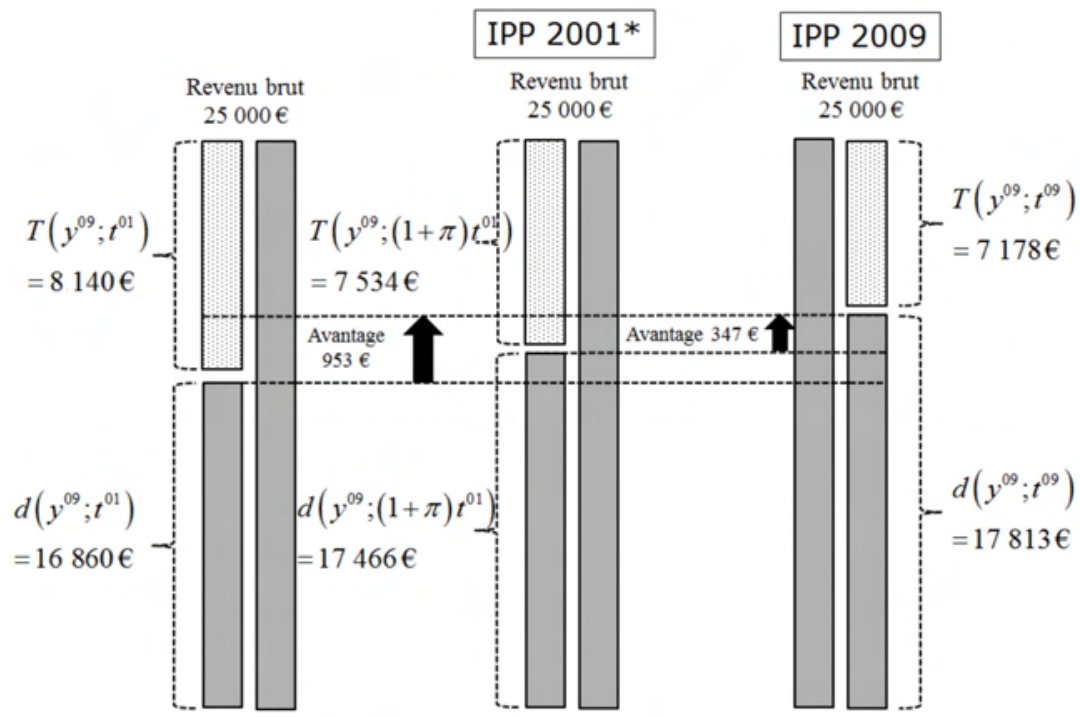

Note : IPP 2001 et IPP 2009 indiquent les règles de calcul de l'impôt sur le revenu de personnes physiques en vigueur en 2001 et 2009, respectivement; IPP 2001* est le contrefactuel des règles en vigueur en 2001.

En considérant une personne qui a un revenu annuel (avant taxes et transferts) de $25000 €$, elle paie $T\left(y^{09} ; t^{09}\right)=-7178 €$ d'impôt direct sur le revenu des personnes en 2009 (IPP 2009 
sur la figure $\left.\mathrm{n}^{\circ} 9\right)$. Si l'on applique l'IPP en vigueur en 2001, elle devrait payer $T\left(y^{09} ; t^{01}\right)=8140 €$. Cependant, une partie importante de la variation est due au fait que les tranches d'imposition sont restées inchangées en termes nominaux (IPP 2001 sur la figure $n^{\circ} 9$ ). Nous ajustons donc les tranches de l'impôt de manière à tenir compte de l'évolution des prix entre 2001 et 2009.

Dans l'exemple représenté sur la figure $\mathrm{n}^{\circ} 9$, nous multiplions les tranches par le facteur d'ajustement pour l'année 2001, $1+\pi=1,195$, c'est-à-dire $1+$ le taux de croissance des prix. Ainsi, le montant total de l'impôt redevable est $T\left(y^{09} ; 1,195 t^{01}\right)=7534 €\left(I P P 2001^{*}\right.$ sur la figure $\mathrm{n}^{\circ}$ 9).

On tirera comme conclusion que sous le régime en vigueur en 2001 cette personne était taxée davantage que sous le régime en vigueur en 2009. Pour résumer, on estime le revenu disponible contrefactuel par application de ces facteurs d'ajustement à toutes les valeurs nominales : $d_{i}\left(\alpha^{i \rightarrow 09} p^{i}, y^{2009}\right)$, avec $1+\pi$ le facteur d'ajustement égal à $1+$ le taux de croissance de l'indice de prix à la consommation entre l'année $i$ et 2009 . 
A. Decoster, S. Perelman, D. Vandelannoote, T. Vanheukelom et G. Verbist 123

\section{Annexe 2}

\section{Adaptation du montant des pensions au bien-être}

\section{Pensions travailleurs salariés}

\begin{tabular}{cccc}
\hline Date & $\begin{array}{c}\text { Année de prise } \\
\text { de la pension }\end{array}$ & Hommes & Femmes \\
\hline 1 jan 2002 & $\ldots-1992$ & 1,010 & 1,010 \\
1 jan 2003 & $\ldots-1992$ & 1,010 & 1,010 \\
& $1993-1995$ & 1,020 & 1,020 \\
1 avr 2004 & 1996 & 1,020 & 1,020 \\
1 sep 2005 & 1997 & 1,020 & 1,020 \\
1 sep 2006 & $1998-1999$ & 1,020 & 1,020 \\
1 sep 2007 & $\ldots-1987$ & 1,020 & 1,020 \\
& $2000-2001$ & 1,020 & 1,020 \\
1 sep 2008 & $1988-2003$ & 1,020 & 1,020 \\
1 juin 2009 & $\ldots-1976$ & 1,020 & 1,020 \\
& $1977-1993$ & $/$ & 1,020 \\
& $1994-2008$ & $/$ & 1,015 \\
1 sep 2009 & 2004 & 1,020 & 1,020 \\
1 sep 2010 & 2005 & 1,020 & 1,020 \\
1 sep 2011 & 2006 & 1,020 & 1,020 \\
& $\ldots-1996$ & 1,0225 & 1,0225 \\
1 nov 2011 & $1996-2010$ & 1,0125 & 1,0125 \\
1 sep 2012 & 2007 & 1,020 & 1,020 \\
\hline
\end{tabular}




\section{Notes}

1. Nous utilisons politique fiscale et système fiscal au sens large de ces termes englobant les taxes, les cotisations et les prestations sociales payées en espèces ; cela correspond à l'anglais " tax-benefit policy ". Dans le cadre de cette étude portant sur la Belgique, du côté des taxes et cotisations sociales sont pris uniquement en considération l'impôt sur le revenu des personnes physiques (IPP) et les cotisations sociales à charge du travailleur.

2. Pour une présentation détaillée du modèle Mefisto (Modelling and Evaluating Flanders' FIscal and Social Tomorrow) et son interaction avec Euromod, voir Decancq et al. [2012]. Euromod (Taxbenefit microsimulation model for the European Union) est le modèle de microsimulation développé par l'Iser (Institute for Social and Economic Research) de l'université de Essex en collaboration avec des équipes des pays-membres. Voir Sutherland [2007] et Lietz et Mantovani [2006], ou visiter le site web http://www. iser.essex.ac.uk/euromod, pour plus d'informations sur Euromod.

3. C'est cette approche qui a été utilisée par Immervoll et al. [2007] pour plusieurs pays européens, et par Adam et Browne [2010], pour le Royaume-Uni.

4. Nous illustrons l'importance de l'indexation des paramètres nominaux $\mathrm{du}$ système dans l'annexe $\mathrm{n}^{\circ} 1$.

5. Hills et al [2014] illustrent les différences de résultats selon que l'on choisit comme indice pour l'évolution du revenu nominal le taux de croissance du salaire nominal ou le taux nominal de croissance du PNB.

6. L'enquête EU-Silc a démarrée en 2005, et la dernière année pour laquelle les données étaient disponibles au moment de la réalisation de cet article, sont celles correspondant à l'année 2010.

7. La décomposition présentée dans l'équation (2) peut aussi être écrite avec l'effet des réformes simulé sur base des revenus et des caractéristiques sociodémographiques correspondant à l'année 0 , ce qui donnerait un résultat et une décompositiion de Shorrocks-Shapley pour obtenir une moyenne de ces mesures.

8. Nos simulations ne tiennent pas compte de l'augmentation de l'âge normal de la pension des femmes, de 60 à 65 ans (d'un an tous les trois ans), entre 1997 et 2007.

9. Voir Lambert [2001] pour plus de détails sur les indices de Kakwani [1977] et de Reynolds et Smolensky [1977].

10. Par souci de simplification de notation, on ne tient pas compte de tous les autres facteurs, notamment des paramètres, intervenant dans le calcul de taxes et de transferts sociaux.

11. Il faut ici faire remarquer que Kleven et Kreiner [2006] développent un cadre d'analyse plus général, basé sur SMCPF pour MCPF "social ", qui tient compte au même temps de l'effet distributif du système fiscal. Néanmoins, de l'avis même des auteurs, ce calcul requiert des informations sur les préférences individuelles pour le bien-être social, par définition subjectives et inobservables.

12. Dans l'absence d'autres adultes dans le ménage du chômeur le taux appliqué est le taux " isolé ». En présence d'autres adultes, c'est soit le taux " ménage " soit le taux " cohabitant " qui est d'application. Si l'une de ces personnes bénéficie de 
revenus d'une activité professionnelle ou de transferts sociaux c'est le taux « cohabitant " qui sera appliqué, autrement ce sera le taux " ménage ".

13. Cette base de données a été validée pour être utilisée par le modèle de microsimulation Mefisto Euromod.

14. Il aurait été souhaitable de prendre aussi en compte l'offre de travail des personnes actives au-delà de l'âge de 59 ans. Cela n'a pas été possible étant donnée la difficulté à identifier ces personnes dans EU-SILC. En effet, dans un nombre important des cas, elles bénéficient au cours de la même année de revenus du travail et de pensions. De plus, les travailleurs âgés constituent un groupe spécifique dont l'offre de travail (marge extensive) est influencée par les règlementations en matière de retraite, non modélisées dans le cadre de ce travail.

15. Il faut signaler que nos simulations sont basées sur l'hypothèse que seule une fraction, estimée à $45 \%$ des personnes potentielles bénéficiaires du revenu minimum garanti (assistance sociale) en bénéficient vraiment. Ces allocations sont assignées par le programme de simulation de manière aléatoire parmi tous les potentiels bénéficiaires.

16. Au cours de la période analysée, les gouvernements successifs ont donné lieu aux coalitions suivantes : Dehaene I (1992-1995) et II (1995-1999) majorité social-chrétienne et socialiste; Verhofstadt I (1999-2003), II (2003-2007) et III (20072008) majorité libérale et socialiste.

17. Etant donné que pour mesurer l'effet des réformes sur l'équité, notre analyse se limite à la population âgée de 19 à 59 ans, l'adaptation au bien-être des pensions publiques n'entrent pas en ligne de compte dans ce calcul.

18. Pour rappel, les allocations de chômage et les allocations de l'assistance sociale ont été réévaluées en termes réels au cours de la période. A tel point que, si l'on considère l'allocation d'assistance à la place de l'allocation de chômage comme le revenu disponible en cas de non-travail, les conclusions restent inchangées.

19. Une fois prise en compte la marge extensive. 


\section{Références}

S. Adam et J. Browne [2010] : Redistribution, Work Incentives and Thirty Years Of UK Tax And Benefit Reform, The Institute for Fiscal Studies.

O. Bargain et T. Callan [2010] : Analysing the Effects of Tax-Benefit Reforms on Income Distribution: A Decomposition Approach, Journal of Economic Inequality, $8(1)$, pp. 1-21.

E. K. Browning [1976] : The Marginal Cost Oo Public Funds, The Journal of Political Economy, pp. 283-298.

E. K. Browning et W.R. Johnson [1984] : The Trade-Off Between Equality and Effciency. The Journal of Political Economy, pp. 175-203.

T. Callan, N. Crilly, C. Keane, J R. Walsh et A. N. Shúilleabháin [2011] : Tax, Welfare and Work Incentives. Budget Perspectives 2012, Retrieved from http://www. esri.ie/UserFiles/publications/WP395/ jacb201155.pdf

B. Cantillon et G. Verbist [1999] : De sociale en fiscale herverdeling in België. CSB Berichten.

T. Clark et A. Leicester [2004] : Inequality and Two Decades of British Tax and Benefit Reform. Fiscal Studies, 25(2), juin, pp. 129-158.

B. Dahlby [1998] : Progressive Taxation and the Social Marginal Cost of Public Funds, Journal of Public Economics, 67(1), pp. 105-122.

B. Dahlby [2008] : The Marginal Cost of Public Funds: Theory and Applications, MIT Press Books.

B. Dahlby [2011] : The Marginal Cost of Public Funds and the Flypaper Effect, Int Tax Public Finance (18), pp. 304-321.
K. Decancq, A. Decoster, K. Spiritus et G. Verbist [2012] : Mefisto: A New Microsimulation Model for Flanders, FLEMOSI discussion paper 14 .

A. Decoster et G. Van Camp [2001] : Redistributive Effects of the Shift from Personal Income Taxes to Indirect Taxes: Belgium 1988-1993, Fiscal studies, 22(1), pp. 79-106.

A. Decoster, M. Gérard et C. Valenduc [2002] : Recettes publiques et politique fiscale, in E. de Callataÿ, La fin du déficit budgétaire - Analyse de l'évolution récente des finances publiques belges (1990-2000) Bruxelles: De Boeck, pp. 107-148.

A. Decoster, M. Gérard et C. Valenduc [2012] : Tax Revenue and Tax Policy - A Decade of Tax Cuts, in E. de Callataÿ, et F. Thys-Clément, The Return of the Deficit - Public Finance in Belgium over 2000-2010, Leuven: Leuven University Press, pp. 95-120.

I. Hansson et C. Stuart [1985] : Tax Revenue and the Marginal Cost of Public Funds in Sweden, Journal of Pubilc Economics (27), pp. 331-353.

J. Hills, A. Paulus, H. Sutherland et I. Tasseva (n.d.) : A Lost Decade? Decomposing the Effects of 2001-2011 Tax-Benefit Policy Changes on the Income Distribution in EU Countries, ImPRovE discussion paper $14 / 03$.

H. Immervoll, H. J. Kleven, C. T. Kreiner et E. Saez [2007] : Welfare Reform in European Countries: a Microsimulation Analysis, The Economic Journal, 117(516), pp. 1-44.

N. Kakwani [1977] : Measurement of Tax Progressivity: An International Comparison, The Economic Journal, 87(345), pp. 71-80. 
H. J. Kleven et C. T. Kreiner [2006] : The Marginal Cost of Public Funds: Hours of Work Versus Labor Force Participation, Journal of Public Economics, 90(10-11), 1955-1973. Retrieved from http://www. sciencedirect.com/science/article/pii/ S0047272706000417

P. Lambert [2001] : The Distribution and Redistribution of Income, Manchester University Press.

C. Lietz et D. Mantovani [2006] : A Short Introduction to Euromod: An Integrated European Tax-Benefit Model, Research in Labor Economics, 25, pp. 1-26.

I. Mayshar [1990]: On Measures of Excess Burden and Their Application, Journal of Public Economics, 43(3), pp. 263-289.

M. Reynolds et E. Smolensky [1977] : Public Expenditures, Taxes and the Distribution of Income: The United States, 1950, 1961, 1970. New York: Academic Press.
E. Saez [2002] : Optimal Income Transfer Programs: Intensive Versus Extensive Labor Supply Responses, The Quarterly Journal of Economics, pp. 1039-1073.

H. Sutherland [2007] : Model 10: Euromod - The Tax-Benefit Microsimulation Model for the European Unio,. in A. Gupta, et A. Harding, Modelling our Future: Population Ageing, Health and Aged Care (International Symposia in Economic Theory and Econometrics, vol. 16), Emerald Group Publishing Limited, pp. 483-488.

F. Vandenbroucke [2012] : The Active Welfare State Revisited, CSB working paper (12/09).

G. Verbist [1999] : Het Huwelijksquotiënt: Kostprijs, Verdeling En Sociale Doelmatigheid, CSB Berichten.

G. Verbist et J. Vanhille [2012] : Simulating Rent Subsidies For Households In Flanders. A Distribution Analysis, Flemosi discussion paper (15). 